\title{
The electric double layer structure around charged spherical interfaces
}

\author{
Zhenwei Yao, Mark J. Bowick and Xu Ma \\ Department of Physics, Syracuse University, Syracuse, New York 13244-1130, USA
}

\begin{abstract}
We derive a formally simple approximate analytical solution to the Poisson-Boltzmann equation for the spherical system via a geometric mapping. Its regime of applicability in the parameter space of the spherical radius and the surface potential is determined, and its superiority over the linearized solution is demonstrated.
\end{abstract}

Charged objects in electrolyte solutions are surrounded by electric double layers (EDL) $\underline{\underline{1}}$. One ionic layer is due to a host of chemical interactions, and the second layer is formed by the excess of oppositely charged ions in the solution, screening the charged objects. The EDL structure is responsible for the stability of colloidal dispersions ${ }^{2}$ and various electrostatic phenomena in biophysical systems ${ }^{3}$. The distribution of the screening potential in EDL is characterized by the Poisson-Boltzmann (PB) equation. In this letter, we will study the EDL structure around charged spherical interfaces, which are ubiquitous in colloidal and biophysical systems ${ }^{4.5}$.

Largely due to its nonlinear nature, analytical solutions to the PB equation are available only for planar $\underline{\underline{6}}$ and cylindrical $\mathbb{l}^{7}$ systems. For a spherical system, the analytical solution to the linearized PB equation is available 2 . Despite a recently proposed analytical series solution $\stackrel{8}{*}$, a formally simple approximate solution is still in demand for studying analytical problems that are based on the screening potential in EDL. Numerical techniques ${ }^{\underline{9}}$ and the Debye-Huckel linearized approximations have long been the only available basic methods to solve the PB equation. Various perturbative solutions have been proposed based on the planar solution $\frac{10}{}$, or the linearized solution $\underline{\underline{11}}$, as the zeroth order approximation. Perturbative methods that start with the planar solution are limited to the regime of large spherical radius, while those which start with the linearized solution work in the weak potential regime. The geometric construction of a formally simple approximate analytical solution that can match both the planar and the linearized solutions is one concern of this letter.

In q:-q symmetric electrolytes, the dimensionless Poisson-Boltzmann equation is 22

$$
\Delta \psi=\sinh \psi
$$

in the natural units $k_{B} T / q$ and the Debye length $\kappa^{-1}=\sqrt{\epsilon k_{B} T /\left(8 \pi n q^{2}\right)} \cdot q$ is the absolute value of the charge of ions. We propose an analytic scheme to yield an approximate solution to the Poisson-Boltzmann equation in a spherical system from the known analytical solution to the planar problem. The planar and spherical systems are connected by 


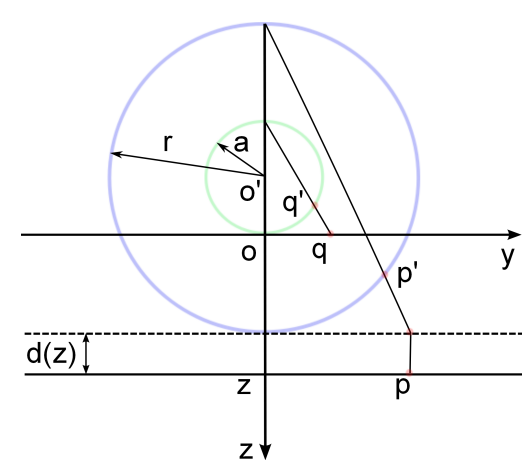

FIG. 1: The mapping from the $z>0$ bulk space to the space outside a sphere with radius $a$ (represented by the blue circle) via. consecutive stereographic projections from a plane to a sphere. A deviation $d(z)$ is introduced to guarantee the equality of the potential on the blue spherical shell and the potential at the plane located at $\mathrm{z}$.

a geometric mapping defined in Fig. 1. Both the planar and spherical systems are schematically plotted in Fig. 11. The planar system is composed of a charged plate locating on the $\mathrm{x}-\mathrm{y}$ plane with the solution in the $z>0$ bulk space; and the corresponding spherical system consists of a charged sphere of radius a centered at the origin, which is immersed in the solution in the $r>a$ bulk space. The surface potential in both systems is denoted as $\psi_{0}$. The potential in the planar system is known as $\psi(z)$, while that around the corresponding spherical system is $\psi(r)$. These two potentials can be related by a geometric mapping which is defined as follows. By moving an arbitrary equipotential plane at $z$ in the planar system by $d(z)$ followed by a stereographic projection as shown in Fig. 1, the equipotential plane $\psi(z)$ in the plate system is geometrically mapped to the equipotential spherical shell $\psi(r(z))$ in the corresponding spherical system. The charged plate on the $\mathrm{x}-\mathrm{y}$ plane is mapped to the spherical interface at $r=a$, so $d(z=0)=0$. The whole $z>0$ bulk space in the planar system can be mapped to the bulk space outside the spherical interface by repeating the mapping defined above for all equipotential planes below the x-y plane.

The displacement field $d(z)$ is introduced to guarantee that

$$
\psi(z)=\psi(r)
$$

with $r=a+z-d(z) . d(z)$ encodes all information of the potential about the spherical interface. Geometrically $d(z)$ describes how the equipotential planes in the planar system squeeze to form the equipotential spherical shells in the corresponding spherical system. The problem for solving the PB equation in a spherical system is now converted to solving for the geometric deviation $d(z)$. The form of $d(z)$ can be found in the weak potential regime where both $\psi_{L}(z)$ of a planar system and $\psi_{L}(r)$ of a spherical system are known:

$$
\psi_{L}(z)=\psi_{0} e^{-z}
$$




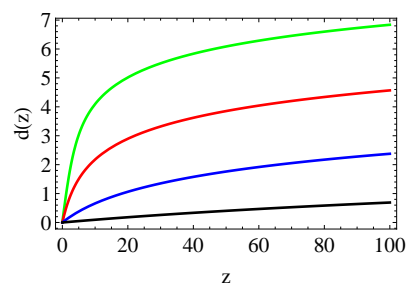

(a)

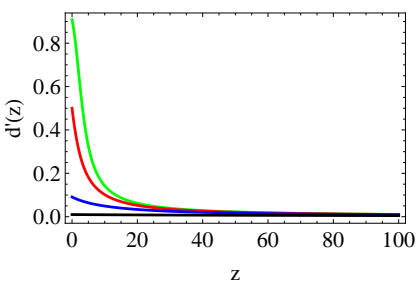

(b)

FIG. 2: (a) The plot of the displacement field $d(z)$ for various spherical radii: $a=0.1$ (green), $a=1$ (red), $a=10$ (blue), $a=100$ (black). As $a \rightarrow \infty, d(z)$ is expected to vanish. (b) The plot of the strain fields $d^{\prime}(z)$ for the corresponding displacement fields $d(z)$ in (a).

and

$$
\psi_{L}(r)=\psi_{0} \frac{a}{r} e^{a-r}
$$

where $a$ is the radius of the spherical interface and the subscript L stands for linearized solution. Inserting Eq. (3) 4) into Eq. (2) leads to $a /(a+z-d(z))=\exp (-d(z))$, from which we have

$$
d(z)=a+z-W\left(a e^{a+z}\right)
$$

where $W(x)$ is the Lambert's W function defined by $x=W(x) \exp (W(x))^{12}$. It is checked that $d(z \rightarrow 0)=0$ and $d(z) \rightarrow 0$ as $a \rightarrow \infty$. And $d(z \rightarrow \infty) \sim \ln (z / a)$, since asymptotically $W(x \rightarrow \infty) \sim \ln x-\ln (\ln x)(\operatorname{Ref} . \quad[12])$ The Lambert's W function is also found in other physical systems, such as the fringe field of a capacitor and Wiens displacement law in black body radiation 13 . Eq. (5) shows that $d(z)$ is independent of $\psi_{0}$ in the weak potential limit, since $\psi_{0}$ appears as a prefactor in both $\psi(z)$ and $\psi(r)$ in the weak potential limit as shown in Eq. (3. 4).

The plot of $d(z)$ for various spherical radii is given in Fig. 2 (a). The squeezing of equipotential surfaces near a spherical interface with smaller radius is seen to be larger. It is expected that the displacement vanishes for an infinitely large spherical interface that approaches a plate. Fig. 2 (a) also shows the behaviors of $d(z)$ in two regions - steep slope for small $z$ and much smaller slope for large $z$ where $d(z \rightarrow \infty) \sim \ln (z / a)$. It gives the qualitative picture of forming equipotential spherical shells from the corresponding equipotential planar slices. Near the charged spherical interface each slice moves more than the slice ahead of it that is closer to the interface. In the region far away from the interface, the squeezing is much more uniform. The relative squeezing of equipotential surfaces is better demonstrated in terms of the "strain" field $d^{\prime}(z)$. The strain field plotted in Fig. 22 (b) shows that the strain is concentrated near the spherical interface and the strain concentration is more significant near spherical interfaces of smaller radii. 


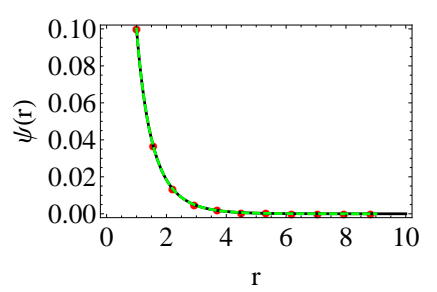

(a)

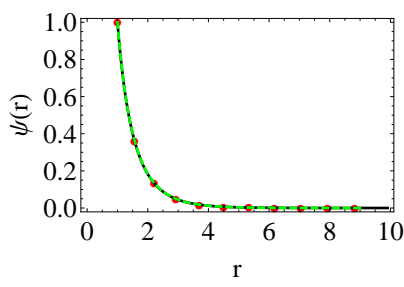

(b)

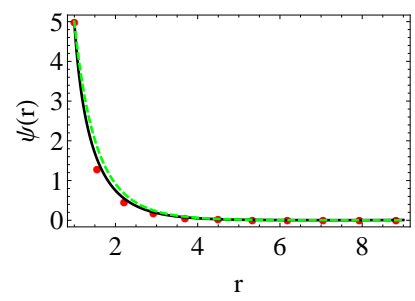

(c)

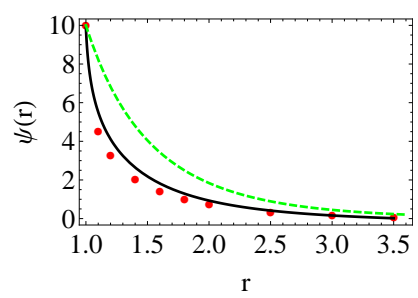

(d)

FIG. 3: The potential around a charged spherical interface of unit radius in a q:q solution. The surface potentials in (a)-(d) are respectively $0.1,1,5,10$ measured by $q /\left(k_{B} T\right) \equiv 1$. The curves give the numerical solution to the Poisson-Boltzmann equation (black), analytical solution for weak $\psi_{0}$ (green dashed) and the solution constructed by the our method (red dots).

We suggest that the form of $d(z)$ for arbitrary $\psi_{0}$ be approximated by Eq. (5) under the assumption of weak dependence of $d(z)$ on $\psi_{0}$. This assumption is to be substantiated later. We can then construct the analytical approximate solution to the $\mathrm{PB}$ equation for a spherical system from the known analytical solution to a planar system, which is

$$
\psi=2 \ln \frac{1+\gamma e^{-z}}{1-\gamma e^{-z}}
$$

where $\gamma=\left(\exp \left(\psi_{0} / 2\right)-1\right) /\left(\exp \left(\psi_{0} / 2\right)+1\right)$. On the other hand, $r(z)=W(a \exp (a+z))$ and $W(a \exp (a+$ $z)) \exp (W(a \exp (a+z)))=a \exp (a+z)$ yield

$$
z(r)=r-a+\ln (r / a)
$$

The approximate solution denoted as $\psi_{G}(r)$ for the spherical system is thus derived as

$$
\psi_{G}(r)=2 \ln \left[\frac{1+\gamma \exp (-(r-a+\ln (r / a)))}{1-\gamma \exp (-(r-a+\ln (r / a)))}\right]
$$

where the RHS is the potential in the corresponding plate system with $z$ replaced by $z(r)$. Near a spherical interface of large radius, i.e., $(r-a) / a<<1$ and $a>>1$, the $\psi_{G}$ solution approaches the planar solution, as required. In the region far away from the interface $(r>>a)$, Eq. (8) becomes $\psi_{G}(r)=4 \gamma a \exp (-(r-a)) / r$, which reduces to the linear spherical solution Eq. (4) in the weak potential limit. Note that the $\psi_{G}$ solution may be derived algebraically by a variable substitution $s=a / r \exp (-(r-a))$ in Eq. (11) and more accurate results can be obtained by perturbation analysis $\frac{14.15}{}$. In comparison to the algebraic method, the derivation of the $\psi_{G}$ solution via the geometric mapping not only reduces the complexity of algebraic calculations, but also shows how the spherical geometry modifies the equipotential surfaces of a planar system as encoded by the geometric deviation $d(z)$. The relation between the $\psi_{G}$ solution and both the linearized and planar solutions is also revealed in the geometric derivation. 
Eq. (8) is derived from the planar and the linearized spherical solution, so at the very least it is expected to work for either $a>>1$ or $\psi_{0}<<1$. It is therefore superior to the linear solution, which only works in the weak potential limit. The region of validity of the $\psi_{G}$ solution can be derived algebraically. By introducing $x=1 / r$, the PB equation for a spherical system becomes 16

$$
C(x) \frac{\partial^{2}}{\partial x^{2}} \psi=\sinh \psi
$$

where $C(x)=x^{4}$. Inspired by the functional form of the planar and the linearized spherical solutions Eqs. (3), 4), we use $\psi(x)=2 \ln ((1+g(x)) /(1-g(x)))$ as a trial solution. Depending on the sign of $\psi(x), g(x)= \pm \exp (-f(x))$ and $g(x) \in(-1,1)$ corresponding to $\psi \in(-\infty, \infty)$. Inserting the ansatz into Eq. (9) yields

$$
g(x)\left[C(x)\left(f^{\prime 2}-f^{\prime \prime}\right)-1\right]+g^{3}\left[C(x)\left(f^{\prime 2}+f^{\prime \prime}\right)-1\right]=0
$$

For $|g|<<1$, by dropping the $g^{3}$ term, the solution to Eq. (10) is $f(x)=1 / x-\ln x+c_{1}$, with an integration constant $c_{1}$. Inserting $f(x)$ into the ansatz $\psi$ yields the $\psi_{G}$ solution. An alternative condition for dropping the $g^{3}$ term in Eq. (10) is $C(x)\left(f^{\prime 2}+f^{\prime \prime}\right)-1<<1$, which is equivalent to $x=1 / r<<1$ by inserting the expressions for $f(x)$ and $C(x)$. Therefore, for either $|g|<<1$ or $x=1 / r<<1$, the solution to Eq. (10) coincides with the $\psi_{G}$ solution. Note that $|g|<<1$ is equivalent to the weak potential limit, and $x=1 / r<<1$ holds for $a>>1$ since $r>a$. An important case falls in this region of validity of the $\psi_{G}$ solution. Consider colloids of size $R$ in a solution of ion strength $I$ (in mol/L). The Debye length is $\kappa^{-1}(\mathrm{~nm})=0.304 / \sqrt{I(\mathrm{~mol} / \mathrm{L})}$ which is at the order of $\mathrm{nm}$ for $I \sim 1 \mathrm{~mol} / \mathrm{L}^{17}$. For $R \sim \mu \mathrm{m}$, $a=R / \kappa^{-1}>>1$. Therefore, the approximate analytical $\psi_{G}$ solution is suitable for typical colloidal dispersions. In comparison to the series solution $\stackrel{8}{*}$, the formal simplicity of the $\psi_{G}$ solution enables further analytical study of the electrostatics of colloidal systems.

The $\psi_{G}$ solution turns out to have a larger region of validity. Fig. 3 shows comparisons of the $\psi_{G}$ solution (red dots), the linearized solution (green dashed) and the numerical solution (black) to the PB equation for a spherical system for different potentials. For weak potential $\left(\psi_{0}=0.1(a), 1(b)\right)$, the three solutions fall on the same curve. The linearized solution works well at least up to $\psi_{0}=5$ without qualitatively deviating from the numerical solution. So the linearized theory applies for moderate values of surface potential18. As $\psi_{0}$ exceeds 5 , the linearized solution starts to deviate from the numerical solution, while $\psi_{G}$ conforms to the numerical solution up to $\psi_{0}=10$, where the linearized solution deviates significantly the numerical solution. This indicates that the dependence of $d(z)$ on $\psi_{0}$ is weak for $a=1$ up to $\psi_{0}=10$.

The quality of the $\psi_{G}$ solution can be systematically studied by defining a ratio $\delta=\max _{r}\{|(\Delta \psi-\sinh \psi) / \sinh \psi|\}$. 


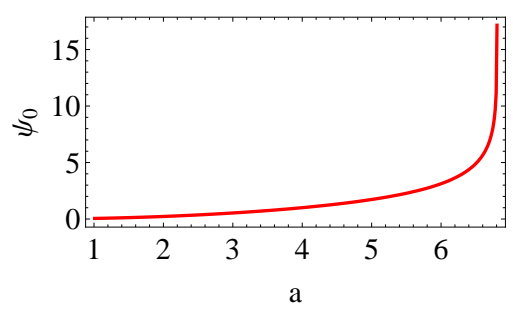

FIG. 4: The $\psi_{G}$ solution is applicable below the red curve in the parameter space $\left\{a, \psi_{0}\right\}$. On the red curve, $\delta=\epsilon=10^{-3}$.

The smaller the ratio $\delta$ is, the better the solution is. For a given precision $\epsilon=10^{-3}$, the applicable region of the $\psi_{G}$ solution is found to be below the red curve in the parameter space $\left\{a, \psi_{0}\right\}$, as shown in Fig. 目 For $a \gtrsim 7$, the $\psi_{G}$ solution applies even for large potentials. There exists, however, a cut-off value for the surface potential. High potential, or equivalently low temperature, may lead to correlation of counter-ions near the charged interface that is ignored in the mean field PB equation $\underline{3}^{\text {. In }}$ addition, high potential leads to high concentration of ions so that the finite dimension of ions must be taken into consideration ${ }^{2}$. The advantage of the $\psi_{G}$ solution over the planar solution is shown explicitly in Fig. 5. The $\psi_{G}$ solution works better than the planar solution even for large spherical radius.

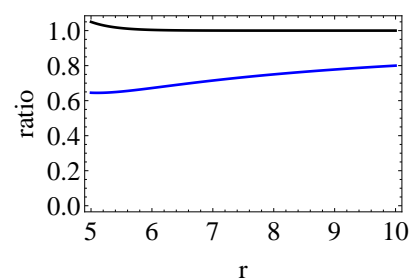

(a)

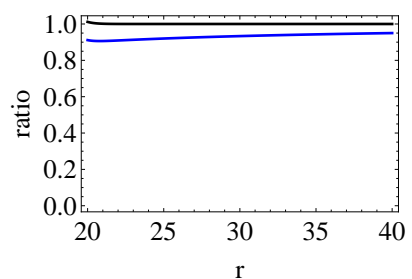

(b)

FIG. 5: The comparison of the $\psi_{G}$ solution (black) and the planar solution (blue) in terms of the ratio of the LHS to RHS of Eq. (1). The radii of the spherical interface are $a=5(a)$ and $20(b) . \psi_{0}=1$.

In conclusion, we have studied the EDL structure around charged spherical interfaces by analysis of the PoissonBoltzmann equation. Despite the point charge assumption of electrolyte ions and the neglect of ion-ion corrections, the PB equation generally works well especially for problems of electrostatic interaction of colloidal particles 1 . In this paper, we have derived an approximate analytical solution to the Poisson-Boltzmann equation for the spherical system by a geometric mapping. The formal simplicity of the $\psi_{G}$ solution enables further analytical study of spherical systems. The regime of applicability includes not only the weak potential regime where the linearized solution also works well, but also the regime of large spherical radius. Typical colloidal dispersions with the size of colloids much bigger than the Debye length fall in the latter regime. 


\section{Acknowledgments}

We thank Shiliyang Xu for discussions. This work was supported by the National Science Foundation grant DMR0808812 and by funds from Syracuse University.

${ }^{1}$ H. Ohshima, Theory of Colloid and Interfacial Electric Phenomena (Academic Press Inc., 2006).

${ }^{2}$ E.J.W. Verwey and J.Th.G. Overbeek, Theory of the stability of lyophobic colloids (Elsevier, 1948).

3 W.M. Gelbart et al., Phys. Today 53, 38 (2000).

${ }^{4}$ W.B. Russel, D.A. Saville and W.R. Schowalter, Colloidal Dispersions (Cambridge University Press, 1992).

${ }^{5}$ B. Honig and A. Nicholls, Science 268, 1144 (1995).

${ }^{6}$ F. Andrietti, A. Peres and R. Pezzotta, Biophys. J. 16(9), 1121 (1976).

7 C.A. Tracy and H. Widom, Physica A 244, 402 (1997).

8 L.G. D'yachkov, Tech. Phys. Lett. 31, 204 (2005).

9 A.L. Loeb, J.Th.G. Overbeek and P.H. Wiersema, The Electrical Double Layer Around a Spherical Particle (M.I. T. Press, Cambridge, Mass., 1961).

10 Jean-Yves Parlange, J. Chem. Phys. 57, 376 (1972).

11 S.L. Brenner and R.E. Roberts, J. Phys. Chem. 77, 2367 (1973).

12 E.W. Weisstein, CRC Concise Encyclopedia of Mathematics, 2nd edition (CRC Press, 2003).

13 S.R. Valluri, D.J. Jeffrey and R.M. Corless, Canad. J. Phys. 78, 823 (2000).

14 L.R. White, J. Chem. Soc. Faraday Trans. 2, 577 (1977).

15 H. Ohshima, T.W. Healy and L.R. White, J. Colloid Interface Sci. 90, 17 (1982).

16 The PB equation can be written in a unified form: $C(x) \psi^{\prime \prime}(x)=\sinh \psi(x)$ in the planar $(C(x)=1$ with $x=z)$, cylindrical $(C(x)=\exp (-2 x)$ with $x=\ln r)$ and spherical $\left(C(x)=x^{4}\right.$ with $\left.x=1 / r\right)$ cases.

17 J. Israelachvili, Intermolecular and Surface Forces (Academic Press Inc., 1985).

18 M. Bier et. al., J. Chem. Phys. 130, 024703 (2009). 